\title{
AN APPLICATION OF A METHOD OF MARCINKIEWICZ TO THE ABSOLUTE SUMMABILITY OF FOURIER SERIES
}

\author{
TAMOTSU TSUCHIKURA
}

(Received September 5,1961)

1. Introduction. Various sufficient conditions are known for the convergence of Fourier series at an assigned point or almost everywhere. For example, the Dini, Lebesgue and other tests for the former case and the Kolmogoroff-Seliverstoff and the Marcinkiewicz theorems for the "almost everywhere" case. On the other hand Marcinkiewicz obtained a theorem of another type, that is, for an integrable function $f(x)$ of period $2 \pi$, if

$$
\int_{0}^{h}|f(x+t)-f(x)| d t=O(|h| / \log |1 / h|)
$$

as $h \rightarrow 0$ for every $x$ in a set $E$, then its Fourier series converges almost everywhere in $E$.

A proof of this theorem is based on a decomposition of the function $f(x)$ into the sum of two functions $\psi(x)$ and $\phi(x)$ which satisfy the Dini and the Kolmogoroff-Seliverstoff conditions for some values of $x$ respectively, and on the localization property of convergence of Fourier series.

In this note we are interested in an application of his method to the absolute summability of Fourier series which has the localization property for suitable summability indices.

2. For the absolute summability the following theorems are known.

THEOERM A ([1]). Let $1 \leqq p \leqq 2$ and let $f(x)$ be of period $2 \pi$ and integrable $L^{p}(0,2 \pi)$. If the series

$$
\sum_{j=0}^{\infty}\left(\int_{\pi / 2^{j+1}}^{\pi / 2^{j}} \frac{\left|\boldsymbol{\varphi}_{x}(t)\right|^{p}}{t} d t\right)^{1 / p}
$$

where $2 \boldsymbol{\phi}_{x}(t)=f(x+t)+f(x-t)-2 f(x)$, is convergent, then the Fourier series of $f$ is summable $|C, 1 / p+\varepsilon|$ at the point $x$ for any $\varepsilon>0$.

This theorem shows that the summability $|C, 1 / p+\varepsilon|$ is of local property for $1 \leqq p \leqq 2$. We note that the convergence of the series (2.1) is easily obtained from each of the next two conditions: 
$49 \dot{2}$

$$
\begin{gathered}
\int_{0}^{\pi} \frac{\left|\boldsymbol{\varphi}_{x}(t)\right|^{p}|\log (1 /|t|)|^{p-1+\delta}}{t} d t<\infty, \\
\int_{0}^{t}\left|\boldsymbol{\varphi}_{x}(u)\right|^{p} d u=O\left(t /(\log 1 /|t|)^{p+\eta}\right) \quad \text { as } t \rightarrow 0
\end{gathered}
$$

where $\delta$ and $\eta$ are some positive numbers.

THEOREM B ([2]). Let the Fourier coefficients of $f(x)$ be $a_{n}, b_{n}$. If the series

$$
\sum_{n=0}^{\infty}\left(\sum_{j=2^{n}+1}^{2^{n+1}}\left(a_{j}^{2}+b_{j}^{2}\right)\right)^{1 / 2}
$$

is convergent, then the Fourier series of $f$ is summable $|C, 1 / 2+\varepsilon|$ almost everywhere for any $\varepsilon>0$.

This theorem is an amelioration of the Wang theorem [3] whose conclusion is the same one as above and the assumption is the convergence of the series

$$
\sum_{n=1}^{\infty}\left(a_{n}^{2}+b_{n}^{2}\right)(\log n)^{1+\delta}
$$

where $\delta>0$. The convergence of the series (2.4) is an easy consequence of the convergence of (2.5). Further we note that if

$$
\boldsymbol{\varphi}_{x}(t)=O\left(1 /(\log 1 /|t|)^{1 / 2+e}\right) \quad \text { as } t \rightarrow 0
$$

uniformly in $x$ for some $\varepsilon>0$, the series (2.5) with suitable $\delta>0$ and $a$ fortiori the series (2.4) are convergent, hence the Fourier series of $f$ is summable $|C, 1 / 2+\varepsilon|$ almost everywhere.

3. We shall prove the following theorem:

THEOREM. Let $f(x)$ be of period $2 \pi$ and integrable $L^{p}(0,2 \pi)(1 \leqq p \leqq 2)$. If

$$
\int_{0}^{h}|f(x+t)-f(x)|^{p} d t=O\left(|h|(\log 1 /|h|)^{-p}(\log \log 1 /|h|)^{1-p+e}\right)
$$

as $h \rightarrow 0$ for $x \in E \subset(0,2 \pi)$ where $\varepsilon$ is some positive number, then the Fourier series of $f$ is summable $|C, 1 / p+\delta|$ almost everywhere in $E$ for any $\delta>0$.

In the case $p=1$ we may take $\varepsilon=0$, that is, (3.1) may be replaced by the weaker condition

$$
\int^{h}|f(x+t)-f(x)| d t=O(|h| / \log 1 /|h|)
$$


PROOF. We shall follow the Marcinkiewicz method (cf. Zygmund [4] p. 170) except the explicit use of the notion of density point.

If the Fourier series of two functions are both absolutely summable, so is the Fourier series of the sum function. Therefore it is enough to show that the function $f$ can be written

$$
f(x)=\phi(x)+\psi(x)
$$

and the absolute summability of Fourier series of $\phi$ is concluded from Theorem B almost everywhere, and that of $\psi$ almost everywhere in $E$ is concluded by Theorem A.

For the simplicity we denote

$$
\begin{aligned}
\lambda(h) & =(\log 1 /|h|)^{-p}(\log 1 /|h|)^{-p+1-\epsilon} & \text { if } 1<p \leqq 2, \\
& =(\log 1 /|h|)^{-1} & \text { if } p=1 .
\end{aligned}
$$

From the assumption we can take a perfect set $P \subset E$ where $|E-P|$ is arbitrarily small and two numbers $M$ and $\eta$ such that

$$
\left|\int_{0}^{h}\right| f(x+t)-\left.f(x)\right|^{p} d t|\leqq M| h \mid \lambda(h) \text { for } x \in P,|h|<\eta .
$$

Then, there exists a constant $M^{\prime}$ such that for any two points $x, y$ in $P$,

$$
|f(x)-f(y)| \leqq M^{\prime} \lambda(h)^{1 / p} \text { where } h=|y-x|<\eta .
$$

In fact, supposing $x<y$ and denoting

$$
\begin{gathered}
\xi(u)=|f(u)-f(x)|^{p} / \lambda(u-x), \\
I=\left(x+\frac{1}{3} h, x+\frac{2}{3} h\right),
\end{gathered}
$$

and $H=[u \in I ; \xi(u)>N]$, we have

$$
\begin{aligned}
|H| & \leqq N^{-1} \int_{I} \xi(u) d u \leqq N^{-1} \lambda(h / 3)^{-1} \int|f(u)-f(x)|^{p} d u \\
& \leqq N^{-1} \lambda(h / 3)^{-1} \int^{h}|f(x+t)-f(x)|^{p} d t \\
& \leqq N^{-1} \lambda(h / 3)^{-1} h \lambda(h) .
\end{aligned}
$$

Since we can find a constant $M_{1}$ independent of $h$ such that $M_{1}>\lambda(h / 3)^{-1} \lambda(h)$, we have

$$
|H| \leqq 3 M_{1}|I| / N
$$

Similarly, considering the function $\zeta(u)=|f(u)-f(y)|^{p} / \lambda(u-y)$ instead of $\xi(u)$ we conclude that the measure of set $H_{1}=[u \in I ; \zeta(u)>N]$ is not greater 
than $3 \cdot M_{2}|I| / N$, where $M_{2}$ is a constant independent of $h$ and $N$. Hence if $N$ is large enough, there exists a point $u_{0} \in I-\left(H \cup H_{1}\right)$ such that

$$
\begin{aligned}
& \left|f(x)-f\left(u_{0}\right)\right| \leqq N^{1 / p} \lambda\left(u_{0}-x\right)^{1 / p}, \\
& \left|f(y)-f\left(u_{0}\right)\right| \leqq N^{1 / p} \lambda\left(y-u_{0}\right)^{1 / p}
\end{aligned}
$$

and we have (3.3) with $M^{\prime}=2 N^{1 / p}$.

Let us put $\phi(x)=f(x)$ for $x \in P$ and let $\phi(x)$ be linear in the closure of each interval contiguous to $P$. Put

$$
\psi(x)=f(x)-\phi(x) .
$$

For $x, y$ in $P$ the function $\phi$ satisfies the condition (3.3) with $\phi$ instead of $f$, and by its linearity in the closure of the contiguous intervals of $P$, we have easily that

$$
|\phi(x)-\phi(y)| \leqq M^{\prime \prime} \lambda(|x-y|)^{1 / p} \quad \text { for }|x-y|<\eta
$$

uniformly in $x$ where $M^{\prime \prime}$ is a constant independent of $x, y$.

Since $1 \leqq p \leqq 2$, we have clearly $\lambda(h)^{1 / p}=O\left(1 /\left(\log 1 /|h|^{1 / 2+\varepsilon}\right)\right.$ as $h \rightarrow 0$, and we see the summability $|C, 1 / 2+\varepsilon|-$ hence $|C, 1 / p+\varepsilon|-$ almost everywhere of the Fourier series of $\phi$ by Theorem B.

Now, in order to prove the summability $|C, 1 / p+\delta|$ almost everywhere in $P$ of Fourier series of $\psi$, we make use of Theorem A. It is sufficient to show that

$$
\sum_{j=0}^{\infty}\left(\int_{x \pm \pi / 2^{\prime}}^{x \pm \pi / 2^{j-1}} \frac{|\psi(t)|^{p}}{|t-x|} d t\right)^{1 / p}<\infty
$$

for almost all $x \in P$. For this purpose we shall only show the finiteness of the following integral

$$
J=\int_{P} d x \sum_{j=0}^{\infty}\left(\int_{x+\pi / 2^{j}}^{x+\pi / 2^{j-1}} \frac{|\psi(t)|^{p}}{|t-x|} d t\right)^{1 / p}
$$

since the other integral in (3.5) is treated similarly. And this fact is the only one left to prove, since we can take $|P|$ so close to $|E|$ as we please.

Denote by $d_{1}=\left(a_{1}, b_{1}\right), d_{2}=\left(a_{2}, b_{2}\right), \ldots$ the intervals contiguous to $P$ and included in $(0,2 \pi)$. For their lengths we use the same letters. We have $\psi(t)=0$ for $t \in P$ and

$$
\begin{aligned}
\int_{a_{t}}^{a_{t}+h} & |\psi(t)|^{p} d t=\int_{a_{t}}^{a_{t}+h}\left|\psi(t)-\psi\left(a_{i}\right)\right|^{p} d t \\
& \leqq 2^{p-1} \int_{a_{\mathfrak{t}}}^{a_{t}+h}\left|f(t)-f\left(a_{i}\right)\right|^{p} d t+2^{p-1} \int_{a_{i}}^{a_{t}+h}\left|\phi(t)-\phi\left(a_{i}\right)\right|^{p} d t
\end{aligned}
$$




$$
\leqq A h \lambda(h)+A h \lambda(h)=A h \lambda(h)
$$

where $A$ is a constant indepent of $h$ and $d_{i}$, but not necessarily the same in each occurrence.

By the Hölder inequality we have

$$
\begin{aligned}
J & \leqq \sum_{j=0}^{\infty}\left(\int_{P} d x \int_{x+\pi / 2^{j}}^{x+\pi \mid 2^{j-1}} \frac{|\psi(t)|^{p}}{|t-x|} d t\right)^{1 / p} \\
& \leqq \sum_{j=0}^{\infty}\left(\int_{0}^{2 \pi}|\psi(t)|^{p} d t \int_{P \cap\left(t-\pi / 2^{j-1}, t-\pi / 2^{j}\right)} \frac{d x}{|t-x|}\right)^{1 / p} \\
& \leqq \frac{1}{\pi} \sum_{j=0}^{\infty} 2^{j / p}\left(\sum_{i=1}^{\infty} \int_{a_{\xi}}|\psi(t)|^{p} d t \int_{P \cap\left(t-\pi / 2^{j-1}, t-\pi / 2^{j}\right)} d x\right)^{1 / p}
\end{aligned}
$$

The inner sum in (3.8) is not greater than

$$
\begin{aligned}
& A \sum_{\substack{i \\
a_{t} \leqq \pi / 2^{j-1}}} \int_{d_{t}}|\psi(t)| \frac{\pi}{2^{j}} d t+A \sum_{\substack{i \\
a_{i}>\pi / 2^{j-1}}} \int_{a_{i}}^{a_{i}+\pi / 2^{j-1}}|\psi(t)|^{p} \frac{\pi}{2^{j}} d t \\
& \quad=S_{1}+S_{2}
\end{aligned}
$$

say, since in the term $S_{2}$, if $a_{i}+\pi / 2^{j-1}<t<b_{i}$, we have $b_{i}>t-\pi / 2^{j}>$ $t-\pi / 2^{j-1}>a_{i}$ and hence $\left(t-\pi / 2^{j-1}, t-\pi / 2^{i}\right) \subset d_{i}$, that is, $P \cap\left(t-\pi / 2^{j-1}\right.$, $t-\pi / 2^{j}$ ) is empty.

To estimate $J$ we divide it into two parts $J_{1}$ and $J_{2}$ corresponding to $S_{1}$ and $S_{1}$ respectively:

$$
J_{1} \leqq A \sum_{j=0}^{\infty} 2^{j / p} S_{1}^{1 / p}, \quad J_{2} \leqq A \sum_{j=0}^{\infty} 2^{j / p} S_{2}^{1 / p} .
$$

Now

$$
J_{1} \leqq A \sum_{j=0}^{\infty}\left(\sum_{i} \int_{d_{t} \leqq \pi_{i} 2^{j-1}}|\psi(t)|^{p} d t\right)^{1 / p} \leqq A \sum_{j=0}^{\infty}\left(\sum_{\substack{i \\ d_{t} \leqq 2_{i} j-1}} d_{i} \lambda\left(d_{i}\right)\right)^{1 / p} .
$$

For any non-negative integer $m$ we denote by $N_{m}$ the number of $i$ such that $\pi / 2^{m}<d_{i} \leqq \pi / 2^{m-1}$. Obviously we have

$$
\sum_{m=0}^{\infty} \frac{N^{m}}{2^{m}} \leqq \frac{1}{\pi} \sum_{i=1}^{\infty} d_{i}<\infty .
$$

Then, (3.9) is less than

$$
A \sum_{j=0}^{\infty}\left(\sum_{\substack{m=j \\ \pi / 2^{m}<d_{i} \leqq \pi / 2^{m-1}}}^{\infty} \sum_{i} \lambda\left(d_{i}\right)\right)^{1 / p}
$$




$$
\leqq A \sum_{j=0}\left(\sum_{m=j}^{\infty} \frac{\pi}{2^{m-1}} \lambda\left(\frac{\pi}{2^{m-1}}\right) N_{m}\right)^{1 / p} \text {. }
$$

In the case $1<p \leqq 2,(3.11)$ is less than

$$
\begin{gathered}
A \sum_{n}^{\infty}\left(\sum_{m=j}^{\infty} \frac{N^{m}}{2^{m}(m+1)^{p}(\log (m+2))^{p-1+\varepsilon}}\right)^{1 / p} \\
\leqq A\left(\sum_{j=0}^{\infty} \frac{\left.\mid \frac{1}{(j+1)(\log (j+2))^{1+\gamma}}\right)^{1 / q}\left(\sum_{j=0}(j+1)^{p-1}(\log (j+2))^{(1+\gamma)(p-1)}\right.}{\left.\times \sum_{m=j}^{\infty} \frac{N_{m}}{2^{m}(m+1)^{p}(\log (m+2))^{p-1+\varepsilon}}\right)^{1 / p}}\right.
\end{gathered}
$$

by use of the Hölder inequality, where $\gamma=\varepsilon /(p-1)$ and $q=p /(p-1)$. The second factor is finite and the last factor is not greater than

$$
\begin{gathered}
A \sum_{m=0}^{\infty} \frac{N_{m}}{2^{m}(m+1)^{p}(\log (m+2))^{p-1+\varepsilon}}(m+1)^{p}(\log (m+2))^{(1+\gamma)(p-1)} \\
\leqq A \sum_{m=0}^{\infty} \frac{N_{m}}{2^{m}}
\end{gathered}
$$

which is finite by (3.10).

In the case $p=1,(3.11)$ is majorated by

$$
A \sum_{j=0}^{\infty} \sum_{m=j}^{\infty} \frac{N_{m}}{2^{m}(m+1)}=A \sum_{m=0}^{\infty} \frac{N_{m}}{2^{m}}<\infty .
$$

Finally we have by (3.7)

$$
\begin{aligned}
& J_{2} \leqq A \sum_{j}^{\infty}\left(\sum_{\substack{i \\
a_{t}>\pi / 2^{j}-1}}^{\infty} \int_{a_{i}}^{a_{i}+\pi / 2^{j-1}}|\psi(t)|^{p} d t\right)^{1 / p} \\
& \leqq A \sum_{j=\underset{n}{n}\left(a_{i}>\pi \mid 2^{j-1}\right.}^{\infty}\left(\sum^{\frac{\pi}{2^{j-1}}} \lambda\left(\frac{\pi}{2^{j-1}}\right)\right)^{1 / p} \\
& \leqq A \sum_{j=0}^{\infty}\left(\frac{1}{2^{j}} \lambda\left(\frac{\pi}{2^{j-1}}\right) \sum_{m=0}^{j-1} N_{m}\right)^{1 / p} .
\end{aligned}
$$

In the case $1<p \leqq 2$, (3.12) is not greaer than

$$
\begin{aligned}
& A \sum_{j=0}^{\infty}\left(\frac{1}{2^{j}(j+1) \log (j+2)} \sum_{m=0}^{-1} N_{m}\right)^{1 / p} \\
& \leqq A\left(\sum_{j=0} \frac{1}{(j+1)(\log (j+2))^{1+\gamma}}\right)^{1 / q}\left(\sum_{j=0}^{\infty} \frac{1}{2^{j}(j+1)} \sum_{m=0}^{j-1} N_{m}\right)^{1 / p}
\end{aligned}
$$


by the Hölder inequality as in $J_{3}$, and (3.13) is $\leqq A \sum N_{m}\left(2^{m}(m+1)\right)^{-1}$ which is finite by (3.10). In the case $p=1,(3.12)$ is less than

$$
A \sum_{j=0}^{\infty} \frac{1}{2^{j}(j+1)} \sum_{m=0}^{j-1} N_{m} \leqq A \sum_{m=0}^{\infty} \frac{N_{m}}{2^{m}(m+1)}<\infty .
$$

Thus we completed the proof of $J<\infty$, and the theorem is proved.

\section{REFERENCES}

[1] KANNO, K. AND TSUCHIKURA, T., On the absolute summability of Fourier series, Tôhoku Math. J.11(1959)456-479.

[2] TsUCHIKURA, T., Absolute summability of Rademacher series, Tôhoku Math. J. 10(1958) 49-59.

[3] WANG, F.T., The absolute Cesàro summability of trigonometrical series, Duke Math. J. 9(1942)567-572.

[4] ZYGMUND, A., Trigonometric Series II, Cambridge (1959).

TÔHOKU UNIVERSITY. 